\title{
101 - Alzheimer Disease and Dementia: diagnostic challenges and future directions in Hispanic populations.
}

Jorge J Llibre Guerra, $M D^{1}$, Daisy Acosta, $M D^{2}$, Ivonne Z. Jiménez Velázquez, $M D^{3}$, Ana RodriguezSalgado, $\mathrm{MSc}^{4}$, Geeske Peeters, $\mathrm{PhD}^{4}$ Juan de Jesús Llibre Rodriguez, MD, PhD ${ }^{5}$.

1. Department of Neurology. Washington University in St. Louis, USA.

2. Universidad Nacional Pedro Henriquez Ureña (UNPHU), Dominican Republic

3. Department of Medicine, University of Puerto Rico, USA.

4. Global Brain Health Institute

5. Havana School of Medicine. La Habana, Cuba.

Alzheimer's disease (AD) and dementia has emerged as a significant societal issue and a global priority. The prevalence of dementia is rising more rapidly in low and middle income countries (LMIC) than in high income countries. A growing body of evidence shows that prevention through risk factor management is the key to reducing the burden of dementia in the society, especially in LMIC. However, a one-size-fits all approach to health promotion is neither efficient nor effective. Latin American countries (LAC) have unique challenges related to dementia, including rapid aging population, high admixture degree and risk factors profile, which influence the prevalence and presentation of dementia. During this session, we will present findings and tools that will help tailor and personalize risk factor management in Hispanics populations. Participants will be first introduced to genetics of Alzheimer disease in Hispanic populations relative to non-Hispanics and the influence of gene by environment interactions. The second presentation will report on the epidemiology and risk factors of AD using cross countries/society comparisons (Non-Hispanics whites vs Hispanics living in US vs Hispanics living in Latin America.) The third presenter will discuss the development of a tool that visualizes how each risk factor contributes to the risk of dementia and how one may lower their risk by addressing the risk factors. The tool can be used in primary care settings in Cuba, Dominican Republic and Puerto Rico. Finally, the fourth presenter uses state-of-the-art digital assessment tools (brain health assessment), for low-cost monitoring of cognitive functioning, $\mathrm{MCl}$ and dementia. Such instruments are important for future evaluation of the impact of preventive strategies. At the end of the presentations, attendees will be able to identify the unique genetic and social determinants that drive AD in LAC. Recommendations will be given for preventive strategies tailored to LMIC. The findings to be shared will be essential for building evidence-based interventions tailored to reducing the burden of dementia in the Hispanic populations.

Session Chair:

Juan Llibre Rodriguez

Alzheimer Disease and genetics in Hispanic Populations

Jorge J Llibre-Guerra

\section{Alzheimer disease epidemiology and risk factors in Caribbean and non-Caribbean Hispanics populations.}

Ivonne Z. Jiménez Velázquez

Development of a tool to motivate for healthy behaviors to prevent dementia in the Caribbean. Daisy Acosta

A brief digital cognitive assessment for detection of cognitive impairment in Hispanics populations. Ana Rodriguez-Salgado 